\title{
An innovative similarity transformation for in-depth research of convection heat and mass transfer
}

\author{
De-Yi Shang ${ }^{1}$, Bu-Xuan Wang ${ }^{2}$, Liang-Cai Zhong ${ }^{3}$ \\ ${ }^{1} 136$ Ingersoll Cres., Ottawa, ON, Canada K2T 0C8 \\ ${ }^{2}$ Dept. of Thermal Engineering, Tsinghua University, Beijing 100084, China \\ ${ }^{3}$ Department of Ferrous Metallurgy, Northeastern University, Shenyang 110004, China
}

\section{Email address:}

deyishang@yahoo.ca (De-Yi S.)

\section{To cite this article:}

De-Yi Shang, Bu-Xuan Wang, Liang-Cai Zhong. An Innovative Similarity Transformation for In-Depth Research of Convection Heat and Mass Transfer. Science Journal of Energy Engineering. Special Issue: Convection Heat and Mass Transfer. Vol. 3, No. 3-1, 2015 , pp. 1-7. doi: 10.11648/j.sjee.s.2015030301.11

\begin{abstract}
Our innovative similarity transformation for in-depth research of convection heat and mass transfer is presented. For solving convection heat and mass transfer issues, the boundary layer analysis method is used, and meanwhile, the FalknerSkan transformation is currently popular to treat the core similarity variables for velocity field similarity. But this type of transformation is inconvenient to do this core work, for similarity transformation of velocity field, because it is necessary to first induce flow function and group theory to derive an intermediate function for an indirect similarity transformation of the velocity field. This case also allows a difficult situation on consideration of variable physical properties. With our innovative similarity transformation, the above inconvenient and difficult situations are avoided, and the velocity components can be directly transformed to the related dimensionless ones. Then, the similarity analysis and transformation of the governing partial differential equations can be simplified greatly. Furthermore, our innovative similarity transformation can conveniently treat variable physical properties and their coupled effect on heat and mass transfer for enhancement of the practical value of convection heat and mass transfer, and so is a better alternative transformation method to the traditional Falkner-Skan transformation. It was proved that the above two innovative methods have a wide practical application in industry.
\end{abstract}

Keywords: Innovative similarity transformation, Convection heat transfer, Boundary layer analysis, Falkner-Skan Transformation, Core similarity variables, Flow function, Group theory

\section{Introduction}

\subsection{Background of Our Innovative Similarity Transformation}

Since Prandtl proposed his famous boundary layer theory [1], many complex issues on convective hydrodynamics and heat and mass transfer could be solved more conveniently. Meanwhile, the similar analysis method is usually used to transform the dimensional physical variables into the dimensionless physical parameters, and the governing partial differential equations are transformed equivalently into the governing ordinary ones. Since the transformed dimensionless parameter is the group of the dimensional physical variables, the transformed governing ordinary differential equation will be greatly simplified. This is the reason the boundary layer theory greatly accelerated the development of the research of convection heat and mass transfer.

Actually, convection heat and mass transfer is strongly affected by fluid variable thermo-physical properties and their interactions within the boundary layer. In fact, the core work of similarity analysis of the governing partial differential equations lies in the similarity transformation of the velocity field of the boundary layer. However, currently popular Falkner-Skan transformation [2] is difficult to do this job, especially with consideration of variable physical properties, because it is necessary to first induce flow function and group theory to derive an intermediate function for an indirect transformation of the velocity field. It is a complicated process, and allows even complicated issue for consideration of variable physical properties. Therefore, most of the related traditional theoretical research on convection heat and mass transfer did not consider or did not 
consider well the coupled effect of variable physical properties. (For more detailed discussion about it, please refer to books $[3,4])$. It lead to that a series of traditional related researches lack practical application value on convection heat and mass transfer, for a simple example, a lot of reported correlations on convection heat and mass transfer application are empirical or semi-empirical obtained from experiment. It is background for development of the innovative similarity transformation.

\subsection{Current Application of the Innovative similarity transformation}

Before introduction of the innovative similarity transformation for in-depth research on convection hydrodynamics and heat and mass transfer, here it is necessary to mention its current application areas. So far, this innovative similarity transformation has been applied in investigation of convection hydrodynamics and heat and mass both without and with phase change, including the following broad areas:

- Free/forced convection [3-7];

- Free film boiling convection $[4,8]$;

- Free/forced film condensation convection of pure vapour $[3,4,9,10]$;

- Free/forced film condensation convection of vapour-gas mixture $[3,4,11]$;

- Accelerating falling film flows of non-Newtonian power law fluids [4, 12-14].

\subsection{Expanded Application of the Innovation similarity transformation}

This innovative similarity transformation has got its wide application so far, since they were proposed on in-depth research for convection hydrodynamics and heat and mass transfer, for example as below:

Studies $[15,16]$ applied our formulization equations on heat transfer coefficient of free convection [4,5] for temperature distribution assessment during radiofrequency ablation related to surgical medical treatment. It is seen that even for the lead phantom close to the edges, for the maximal discrepancy from their test data, a satisfactory agreement $(2 \%)$ was achieved. Our formulization equations on heat transfer coefficient were just obtained by using the present innovative similarity transformation.

Our innovative similarity transformation was applied for study on deposition of $\mathrm{SiO} 2$ nanoparticles in heat exchanger during combustion of biogas [17]. In this study, our set of dimensionless similarity physical parameters with their formulae including our innovative core similarity variables for similarity transformation of velocity field [3] were applied for similarity transformation of the governing partial differential equations of a laminar forced convection. Based on our innovative similarity transformation, the governing ordinary differential equations were obtained for numerical solution.

Ref. [18] successfully applied our heat transfer equations of free convection of gas with consideration of variable physical properties [4,5] for analytical and experimental study on multiple fire sources in a kitchen. It follows that our formulization equation of Nusselt number combined with the gaseous temperature parameter has wide application value for evaluation of heat transfer with large temperature differences. Our formulization equations on heat transfer coefficient were just obtained by using the present innovative similarity transformation.

The above examples also reflect that our innovative method has special theoretical and practical value and broad application prospects in more industrial departments.

\section{The Innovative Similarity Transformation}

Our innovative similarity transformation was originally developed for extensive investigation of coupled effect of temperature- dependent physical properties of gases and liquids on free/forced convection heat transfer [4-7]. The detailed theoretical analysis and mathematical derivation for this transformed of the governing differential equations are reported in our books $[3,4]$. Due to the space limitation here we have to introduce this transformation briefly through its application. Here, free and forced convection boundary layer issues are taken as examples for introduction of our innovative similarity transformation. First, it is necessary to show its governing partial differential equations.

\subsection{For Free Convection Boundary Layer}

\subsubsection{Governing Partial Differential Equations}

Governing partial differential equations of free convection boundary layer with consideration of variable physical properties are

$$
\begin{gathered}
\frac{\partial}{\partial x}\left(\rho w_{x}\right)+\frac{\partial}{\partial y}\left(\rho w_{y}\right)=0 \\
\rho\left(w_{x} \frac{\partial w_{x}}{\partial x}+w_{y} \frac{\partial w_{x}}{\partial y}\right)=\frac{\partial}{\partial y}\left(\mu \frac{\partial w_{x}}{\partial y}\right)+g\left|\rho_{\infty}-\rho\right| \\
\rho c_{p}\left(w_{x} \frac{\partial t}{\partial x}+w_{y} \frac{\partial t}{\partial y}\right)=\frac{\partial}{\partial y}\left(\lambda \frac{\partial t}{\partial y}\right)
\end{gathered}
$$

where Eqs.(1) to (3) are mass, momentum, and energy conservation equations respectively. Here, it is seen that the effect of variable thermo-physical properties, such as density, absolute viscosity, and thermal conductivity are considered. However, for most of common gases and liquids, the variation of specific heat with temperature can be omitted.

\subsubsection{Similarity Physical Variables (or Parameters)}

The detailed physical analysis and mathematical derivation about our innovative similarity analysis method are reported in [4-7] for treatment of above free convection boundary layer. For saving space, here we only show the set of the related dimensionless similarity physical variables (or parameters). However, the core work for the similarity 
analysis and transformation issues is to treat the similarity problem of velocity field. To this end, we proposed the following innovative core similarity variables $W_{x}(\eta)$ and $W_{y}(\eta)$ and their formulae as follows for similarity transformation of velocity field :

$$
\begin{gathered}
W_{x}(\eta)=\left[\left.2 \sqrt{g x}\left|\frac{\rho_{\infty}}{\rho_{w}}-1\right|\right|^{\frac{1}{2}}\right]^{-1} w_{x} \\
W_{y}(\eta)=\left[2 \sqrt{g x}\left|\frac{\rho_{\infty}}{\rho_{w}}-1\right|^{\frac{1}{2}}\left(\frac{1}{4} G r_{x, \infty}\right)^{-\frac{1}{4}}\right]^{-1} w_{y}
\end{gathered}
$$

where the local Grashof number is defined as

$$
\mathrm{Gr}_{\mathrm{x}, \infty}=\frac{\mathrm{g}\left|\rho_{\infty} / \rho_{\mathrm{w}}-1\right| \mathrm{x}^{3}}{\mathrm{v}_{\infty}{ }^{2}}
$$

The similarity coordinate variable is defined as

$$
\eta=\frac{y}{x}\left(\frac{1}{4} G r_{x, \infty}\right)^{1 / 4}
$$

The similarity temperature is given as

$$
\theta=\frac{t-t_{\infty}}{t_{w}-t_{\infty}}
$$

Here, innovative equations $(4,5)$ for similarity velocity variables are derived in our book [4]. It is emphasized that the similarity velocity variables $W_{x}(\eta)$ and $W_{y}(\eta)$ are core similarity variables for similarity transformation of velocity field. Then, it is expected that they will dominate the transformed governing ordinary differential equations.

\subsubsection{Governing Similarity Ordinary Differential Equations}

The whole similarity transformation process for governing similarity ordinary differential equations of free convection boundary layer is reported in our book [4]. Here, we only give the transformed governing ordinary differential equations as below to save space:

$$
\begin{aligned}
& 2 W_{x}(\eta)-\eta \frac{d W_{x}(\eta)}{d \eta}+4 \frac{d W_{y}(\eta)}{d \eta}-\frac{1}{\rho} \frac{d \rho}{d \eta}\left(\eta W_{x}(\eta)-4 W_{y}(\eta)\right)=0 \\
& \frac{v_{\infty}}{v}\left(\mathrm{~W}_{\mathrm{x}}(\eta)\left(2 \mathrm{~W}_{\mathrm{x}}(\eta)-\eta \frac{\mathrm{dW}_{\mathrm{x}}(\eta)}{\mathrm{d} \eta}\right)+4 \mathrm{~W}_{\mathrm{y}}(\eta) \frac{\mathrm{dW}_{\mathrm{x}}(\eta)}{\mathrm{d} \eta}\right) \\
&= \frac{\mathrm{d}^{2} \mathrm{~W}_{\mathrm{x}}(\eta)}{\mathrm{d} \eta^{2}}+\frac{1}{\mu} \frac{\mathrm{d} \mu}{\mathrm{d} \eta} \frac{\mathrm{dW}}{\mathrm{d} \eta}+\frac{\mathrm{v}_{\infty}}{v} \frac{\frac{\rho_{\infty}}{\rho}-1}{\rho_{\infty}}-1 \\
& \operatorname{Pr} \frac{v_{\infty}}{v}\left(-\eta W_{x}(\eta)+4 W_{y}(\eta)\right) \frac{d \theta}{d \eta}=\frac{1}{\lambda} \frac{d \lambda}{d \eta} \frac{d \theta}{d \eta}+\frac{d^{2} \theta}{d \eta^{2}}
\end{aligned}
$$

It is found that the dimensionless velocity similarity variables $W_{x}$ and $W_{y}$ as the core similarity variables dominate the transformed governing ordinary differential equations.

\subsection{For Forced Convection Boundary Layer}

\subsubsection{Governing Partial Differential Equations}

Our innovative similarity transformation for forced convection boundary layer was proposed in [3]. The governing partial differential equations are as follows for consideration of variable thermo-physical properties:

$$
\begin{gathered}
\frac{\partial}{\partial x}\left(\rho w_{x}\right)+\frac{\partial}{\partial y}\left(\rho w_{y}\right)=0 \\
\rho\left(w_{x} \frac{\partial w_{x}}{\partial x}+w_{y} \frac{\partial w_{x}}{\partial y}\right)=\frac{\partial}{\partial y}\left(\mu \frac{\partial w_{x}}{\partial y}\right) \\
\rho c_{p}\left(w_{x} \frac{\partial t}{\partial x}+w_{y} \frac{\partial t}{\partial y}\right)=\frac{\partial}{\partial y}\left(\lambda \frac{\partial t}{\partial y}\right)
\end{gathered}
$$

where Eqs.(12) to (14) are mass, momentum, and energy conservation equations respectively. Here, it is seen that the effect of variable thermo-physical properties, such as density, absolute viscosity, and thermal conductivity are considered. However, for most of common gases and liquids, the variation of specific heat with temperature can be omitted.

\subsubsection{Similarity Physical Variables (or Parameters)}

The detailed physical analysis and mathematical derivation of our innovative similarity analysis method are reported in [3] for similarly equivalent transformation of forced boundary layer convection. For saving space, here we only show the set of equations with the related dimensionless similarity physical variables (or parameters). However, the core work for the similarity analysis and transformation issues is to treat the similarity transformation of velocity field. To this end, we proposed the innovative core similarity variables and their formulae as below for the similarity transformation of the velocity field [3]:

$$
\begin{gathered}
W_{x}(\eta)=\frac{w_{x}}{w_{x, \infty}} \\
W_{y}(\eta)=\left(\frac{1}{2} \operatorname{Re}_{x, \infty}\right)^{1 / 2} \frac{w_{y}}{w_{x, \infty}}
\end{gathered}
$$

where the local Reynolds number is defined as

$$
\operatorname{Re}_{x, \infty}=\frac{w_{x, \infty} x}{v_{\infty}}
$$

The similarity coordinate variable is defined as

$$
\eta=\frac{y}{x}\left(\frac{1}{2} \operatorname{Re}_{x, \infty}\right)^{1 / 2}
$$

The similarity temperature is given as

$$
\boldsymbol{\theta}=\frac{t-t_{\infty}}{t_{w}-t_{\infty}}
$$


Here, Innovative equations $(15,16)$ for similarity transformation of velocity field are originally proposed and derived in our book [3]. It is emphasized that the similarity velocity variables $W_{x}(\eta)$ and $W_{y}(\eta)$ are core similarity variables for whole similarity transformation. Obviously, they will dominate the transformed governing ordinary differential equations.

\subsubsection{Governing transformed Ordinary Differential Equations}

The whole similarity transformation process for governing similarity ordinary differential equations is presented in our book [3]. Here, we only give the transformed governing ordinary differential equations as below:

$$
\begin{gathered}
-\eta \frac{d W_{x}(\eta)}{d \eta}+2 \frac{d W_{y}(\eta)}{d \eta}+\frac{1}{\rho} \frac{d \rho}{d \eta}\left[-\eta \cdot W_{x}(\eta)+2 W_{y}(\eta)\right]=0 \\
\frac{v_{\infty}}{v}\left(-\eta W_{x}(\eta)+2 W_{y}(\eta)\right) \frac{d W_{x}(\eta)}{d \eta}=\frac{d^{2} W_{x}(\eta)}{d \eta^{2}}+\frac{1}{\mu} \frac{d \mu}{d \eta} \frac{d W_{x}(\eta)}{d \eta} \\
\operatorname{Pr} \frac{v_{\infty}}{v}\left(-\eta W_{x}+2 W_{y}\right) \frac{d \theta}{d \eta}=\frac{1}{\lambda} \frac{d \lambda}{d \eta} \frac{d \theta}{d \eta}+\frac{d^{2} \theta}{d \eta^{2}}
\end{gathered}
$$

It is found that the core similarity variables $W_{x}(\eta)$ and $W_{y}(\eta)$ for similarity transformation of velocity field dominate the whole transformed governing ordinary differential equations.

\section{Extensive Application of the Innovative Similarity Transformation}

Since the innovative similarity transformation was developed for hydrodynamics and heat transfer investigation of free and forced convection, its application has been extended to our study on convection hydrodynamics and heat and mass transfer with film boiling/condensation convection, such as free film boiling convection [4,8], free/forced film condensation convection of pure vapour $[3,4,9,10]$, free/forced film condensation convection of vapour-gas mixture $[3,4,11]$. It is indicated that in all of these studies, the complicated physical factors including the coupled effect of variable physical properties were well considered, then, the heat and mass transfer results demonstrate their practical value. To save space, here we only introduce briefly the application of our innovative similarity transformation in extensive study on free film condensation convection of vapour-gas mixture as below:

\subsection{Governing Partial Differential Equations for Free Film Condensation Convection of Vapour-Gas Mixture}

Free film condensation convection of vapour-gas mixture is attributed to multiple phase-film convection. It consists of three parts: condensate liquid film flow, vapour-gas film flow, and the interface with multiple physical balance conditions. The detailed derivation of the related similarity equations of free convection condensation of vapour-gas mixture are reported in our book [4]. For saving space, the interfacial boundary condition equations are omitted, and only the governing partial differential equations of condensate liquid film flow and vapour-gas mixture film flow are described as below with consideration of couples effect of variable thermo-physical properties:

For condensate liquid film flow

$$
\begin{gathered}
\frac{\partial}{\partial \mathrm{x}}\left(\rho_{1} \mathrm{w}_{\mathrm{xl}}\right)+\frac{\partial}{\partial \mathrm{y}}\left(\rho_{1} \mathrm{w}_{\mathrm{yl}}\right)=0 \\
\rho_{l}\left(w_{x l} \frac{\partial w_{x l}}{\partial x}+w_{y l} \frac{\partial w_{x l}}{\partial y}\right)=\frac{\partial}{\partial y}\left(\mu_{l} \frac{\partial w_{x l}}{\partial y}\right)+g\left(\rho_{l}-\rho_{m, \infty}\right) \\
\rho_{l}\left[w_{x} \frac{\partial\left(c_{p_{l}} t\right)}{\partial x}+w_{y} \frac{\partial\left(c_{p_{l}} t\right)}{\partial y}\right]=\frac{\partial}{\partial y}\left(\lambda_{l} \frac{\partial t}{\partial y}\right)
\end{gathered}
$$

where Eqs.(23)-(25) are continuity, momentum and energy conservation equations respectively.

For vapor-gas mixture film

$$
\frac{\partial}{\partial x}\left(\rho_{m} w_{x m}\right)+\frac{\partial}{\partial y}\left(\rho_{m} w_{y m}\right)=0
$$

$$
\begin{array}{r}
\rho_{m}\left(w_{x m} \frac{\partial w_{x m}}{\partial x}+w_{y m} \frac{\partial w_{x m}}{\partial y}\right)=\frac{\partial}{\partial y}\left(\mu_{m} \frac{\partial w_{x m}}{\partial y}\right)+g\left(\rho_{m}-\rho_{m, \infty}\right) \\
\rho_{\mathrm{m}} \mathrm{c}_{\mathrm{p}_{\mathrm{m}}}\left(\mathrm{w}_{\mathrm{xm}} \frac{\partial \mathrm{t}_{\mathrm{m}}}{\partial \mathrm{x}}+\mathrm{w}_{\mathrm{yv}} \frac{\partial \mathrm{t}_{\mathrm{m}}}{\partial \mathrm{y}}\right) \\
=\frac{\partial}{\partial \mathrm{y}}\left(\lambda_{\mathrm{m}} \frac{\partial \mathrm{t}_{\mathrm{m}}}{\partial \mathrm{y}}\right)+\frac{\partial}{\partial \mathrm{y}}\left[\rho_{\mathrm{m}} \mathrm{D}_{\mathrm{v}}\left(\mathrm{c}_{\mathrm{pv}}-\mathrm{c}_{\mathrm{pg}}\right) \frac{\partial \mathrm{C}_{\mathrm{mv}}}{\partial \mathrm{y}} \mathrm{t}_{\mathrm{m}}\right] \\
\frac{\partial\left(w_{x m} \rho_{m} C_{m v}\right)}{\partial x}+\frac{\partial\left(w_{y m} \rho_{m} C_{m v}\right)}{\partial y}=\frac{\partial}{\partial y}\left(D_{v} \rho_{m} \frac{\partial C_{m v}}{\partial y}\right)
\end{array}
$$

where Eqs.(26)-(28) are continuity, momentum and energy conservation equations respectively, while Eq.(29) is species conservation equation.

\subsection{Dimensionless Similarity Parameters and Variables}

Based on our innovative similarity trsmdformation, a set of equations for similarity analysis and transformation are described as below:

For condensate liquid film flow

For convection issue, the core work for the similarity analysis and transformation is always the similarity of velocity field. Then, the core similarity variables and their formulae are as follows for the similarity analysis and transformation of the velocity field [4]:

$$
\begin{gathered}
W_{x l}\left(\eta_{l}\right)=\left(2 \sqrt{g x}\left(\frac{\rho_{l, w}-\rho_{m, \infty}}{\rho_{l, s}}\right)^{1 / 2}\right)^{-1} w_{x l} \\
W_{y l}\left(\eta_{l}\right)=\left(2 \sqrt{g x}\left(\frac{\rho_{l, w}-\rho_{m, \infty}}{\rho_{l, s}}\right)^{1 / 2}\left(\frac{1}{4} G r_{x l, s}\right)^{-1 / 4}\right)^{-1} w_{y l}
\end{gathered}
$$

In addition, the local Grashof number $G r_{x l, s}$ is set up as 


$$
G r_{x l, s}=\frac{g\left(\rho_{l, w}-\rho_{m, \infty}\right) x^{3}}{{v_{l, s}}^{2} \rho_{l, s}}
$$

The Dimensionless coordinate variables $\eta_{l}$ is set up as follows:

$$
\eta_{l}=\left(\frac{1}{4} G r_{x l, s}\right)^{1 / 4} \frac{y}{x}
$$

Dimensionless temperature is assume as

$$
\theta_{l}=\frac{t_{l}-t_{s, \mathrm{int}}}{t_{w}-t_{s, \mathrm{int}}}
$$

For vapour-gas mixture film flow

In view of that the core work for the similarity analysis and transformation is always to treat the similarity problem of velocity field for convection issue, based on our innovative similarity transformation, we proposed the following innovative dimensionless similarity variables and their formulae for the similarity treatment of the velocity field of vapour-gas mixture film flow [4]:

$$
\begin{gathered}
W_{x m}\left(\eta_{l}\right)=\left(2 \sqrt{g x}\left(\rho_{m, s} / \rho_{m, \infty}-1\right)^{1 / 2}\right)^{-1} w_{x m} \\
W_{y m}\left(\eta_{l}\right)=\left(2 \sqrt{g x}\left(\rho_{m, s} / \rho_{m, \infty}-1\right)^{1 / 2}\left(\frac{1}{4} G r_{x m, \infty}\right)^{-1 / 4}\right)^{-1} w_{y m}
\end{gathered}
$$

Additionally, the local Grashof number $G r_{x m, \infty}$ of vapourgas mixture film is set up as

$$
G r_{x m, \infty}=\frac{g\left(\rho_{m, s} / \rho_{m, \infty}-1\right) x^{3}}{v_{m, \infty}^{2}}
$$

The dimensionless coordinate variables $\eta_{m}$ of vapour-gas mixture film flow is set up as

$$
\eta_{m}=\left(\frac{1}{4} G r_{x m, \infty}\right)^{1 / 4} \frac{y}{x}
$$

The dimensionless temperature of vapour-gas mixture flow film is defined as

$$
\theta_{m}=\frac{t_{m}-t_{\infty}}{t_{s, \text { int }}-t_{\infty}}
$$

The vapor relative mass fraction is defined as

$$
\Gamma_{m v}\left(\eta_{m}\right)=\frac{C_{m v}-C_{m v, \infty}}{C_{m v, s}-C_{m v, \infty}}
$$

\subsection{Governing Ordinary Differential Equations}

By means of Eqs.(30) to (34), the governing partial differential equations (23) to (25) are equivalently transformed to the following ordinary partial equations:

\subsubsection{For Liquid Film Flow}

$$
\begin{gathered}
2 \mathrm{~W}_{\mathrm{xl}}\left(\eta_{1}\right)-\eta_{1} \frac{\mathrm{dW}_{\mathrm{xl}}\left(\eta_{1}\right)}{\mathrm{d} \eta_{1}}+4 \frac{\mathrm{dW}_{\mathrm{yl}}\left(\eta_{1}\right)}{\mathrm{d} \eta_{1}} \\
-\frac{1}{\rho_{1}} \frac{\mathrm{d} \rho_{1}}{\mathrm{~d} \eta_{1}}\left(\eta_{1} \mathrm{~W}_{\mathrm{xl}}\left(\eta_{1}\right)-4 \mathrm{~W}_{\mathrm{yl}}\left(\eta_{1}\right)\right)=0 \\
\frac{v_{l, s}}{v_{l}}\left[W_{x l}\left(\eta_{l}\right)\left(2 W_{x l}\left(\eta_{l}\right)-\eta_{l} \frac{d W_{x l}\left(\eta_{l}\right)}{d \eta_{l}}\right)+4 W_{y l}\left(\eta_{l}\right) \frac{d W_{x l}\left(\eta_{l}\right)}{d \eta_{l}}\right] \\
=\frac{d^{2} W_{x l}\left(\eta_{l}\right)}{d \eta_{l}^{2}}+\frac{1}{\mu_{l}} \frac{d \mu_{l}}{d \eta_{l}} \frac{d W_{x l}\left(\eta_{l}\right)}{d \eta_{l}}+\frac{\mu_{l, s}}{\mu_{l}} \frac{\left(\rho_{l}-\rho_{m, \infty}\right)}{\rho_{l, w}-\rho_{m, \infty}} \\
\operatorname{Pr}_{l} \frac{v_{l, s}}{v_{l}}\left(-\eta_{l} W_{x l}\left(\eta_{l}\right)+4 W_{y l}\left(\eta_{l}\right)\right) \frac{d \theta_{l}}{d \eta_{l}}=\frac{d^{2} \theta_{l}}{d \eta_{l}^{2}}+\frac{1}{\lambda_{l}} \frac{d \lambda_{l}}{d \eta_{l}} \frac{d \theta_{l}}{d \eta_{l}}
\end{gathered}
$$

where Eqs.(41)-(43) are continuity, momentum and energy conservation equations respectively (see our book [4] for the detailed mathematical derivation). Here, it is seen that the core similarity variables, dimensionless similarity velocity variables dominate the governing ordinary differential equations of condensate liquid film.

\subsubsection{For Vapour-Gas Mixture Film Flow}

By means of Eqs.(35) to (40), the governing partial differential equations (26) to (29) are equivalently transformed to the following ordinary partial equations respectively:

$$
\begin{aligned}
& 2 \mathrm{~W}_{\mathrm{xm}}\left(\eta_{\mathrm{m}}\right)-\eta_{\mathrm{m}} \frac{\mathrm{dW_{xm }}\left(\eta_{\mathrm{m}}\right)}{\mathrm{d} \eta_{\mathrm{m}}}+4 \frac{\mathrm{dW}_{\mathrm{ym}}\left(\eta_{\mathrm{m}}\right)}{\mathrm{d} \eta_{\mathrm{m}}} \\
& -\frac{1}{\rho_{\mathrm{m}}} \frac{\mathrm{d} \rho_{\mathrm{m}}}{\mathrm{d} \eta_{\mathrm{m}}}\left(\eta_{\mathrm{m}} \mathrm{W}_{\mathrm{xm}}\left(\eta_{\mathrm{m}}\right)-4 \mathrm{~W}_{\mathrm{ym}}\left(\eta_{\mathrm{m}}\right)\right)=0 \\
& \frac{v_{m, \infty}}{v_{m}}\left[W_{x m}\left(\eta_{m}\right)\left(2 W_{x m}\left(\eta_{m}\right)-\eta_{m} \frac{d W_{x m}\left(\eta_{m}\right)}{d \eta_{m}}\right)+4 W_{y m}\left(\eta_{m}\right) \frac{d W_{x m}\left(\eta_{m}\right)}{d \eta_{m}}\right] \\
& =\frac{d^{2(\eta)} W_{x m}\left(\eta_{m}\right)}{d \eta_{m}^{2}}+\frac{1}{\mu_{m}} \frac{d \mu_{m}}{d \eta_{m}} \frac{d W_{x m}\left(\eta_{m}\right)}{d \eta_{m}}+\frac{\mu_{m, \infty}}{\mu_{m}} \cdot \frac{\rho_{m}-\rho_{m, \infty}}{\rho_{m, s}-\rho_{m, \infty}} \\
& \frac{v_{m, \infty}}{v_{m}}\left[-\eta_{m} W_{x m}\left(\eta_{m}\right)+4 W_{y m}\left(\eta_{m}\right)\right] \frac{d \theta_{m}}{d \eta_{m}} \\
& =\frac{1}{\operatorname{Pr}_{\mathrm{m}}}\left(\frac{\mathrm{d}^{2} \theta_{\mathrm{m}}}{\mathrm{d} \eta_{\mathrm{m}}{ }^{2}}+\frac{1}{\lambda_{\mathrm{m}}} \frac{\mathrm{d} \lambda_{\mathrm{m}}}{\mathrm{d} \eta_{\mathrm{m}}} \cdot \frac{\mathrm{d} \theta_{\mathrm{m}}}{\mathrm{d} \eta_{\mathrm{m}}}\right) \\
& -\frac{1}{\mathrm{Sc}_{\mathrm{m}, \infty}} \frac{\mathrm{v}_{\mathrm{m}, \infty}}{v_{\mathrm{m}}}\left(\mathrm{C}_{\mathrm{mv}, \mathrm{s}}-\mathrm{C}_{\mathrm{mv}, \infty}\right) \frac{\mathrm{c}_{\mathrm{pv}}-\mathrm{c}_{\mathrm{pg}}}{\mathrm{c}_{\mathrm{p}_{\mathrm{m}}}} \\
& {\left[\frac{\mathrm{d} \theta_{\mathrm{m}}}{\mathrm{d} \eta_{\mathrm{m}}} \frac{\mathrm{d} \Gamma_{\mathrm{mv}}\left(\eta_{\mathrm{m}}\right)}{\mathrm{d} \eta_{\mathrm{m}}}+\left(\theta_{\mathrm{m}}+\frac{\mathrm{t}_{\infty}}{\mathrm{t}_{\mathrm{s}, \mathrm{int}}-\mathrm{t}_{\infty}}\right) \frac{\mathrm{d}^{2} \Gamma_{\mathrm{mv}}\left(\eta_{\mathrm{m}}\right)}{\mathrm{d} \eta_{\mathrm{v}}{ }^{2}}\right.} \\
& \left.+\left(\theta_{\mathrm{m}}+\frac{\mathrm{t}_{\infty}}{\mathrm{t}_{\mathrm{s}, \mathrm{int}}-\mathrm{t}_{\infty}}\right) \frac{1}{\rho_{\mathrm{m}}} \frac{\mathrm{d} \rho_{\mathrm{m}}}{\mathrm{d} \eta_{\mathrm{m}}} \frac{\mathrm{d} \Gamma_{\mathrm{mv}}\left(\eta_{\mathrm{m}}\right)}{\mathrm{d} \eta_{\mathrm{m}}}\right] \\
& {\left[-\eta_{\mathrm{m}} \mathrm{W}_{\mathrm{xm}}\left(\eta_{\mathrm{m}}\right)+4 \mathrm{~W}_{\mathrm{ym}}\left(\eta_{\mathrm{m}}\right)\right] \frac{\mathrm{d} \Gamma_{\mathrm{m}, \mathrm{v}}\left(\eta_{\mathrm{m}}\right)}{\mathrm{d} \eta_{\mathrm{m}}}} \\
& =\frac{1}{S c_{m, \infty}}\left[\frac{d^{2} \Gamma_{m, v}\left(\eta_{m}\right)}{d \eta_{v}^{2}}+\frac{1}{\rho_{m}} \frac{d \rho_{m}}{d \eta_{m}} \frac{d \Gamma_{m, v}\left(\eta_{m}\right)}{d \eta_{m}}\right]
\end{aligned}
$$

where Eqs.(44)-(46) are continuity, momentum, and energy conservation equations, while Eq.(47) is the species 
conservation equation with mass diffusion (see our book [4] for the detailed mathematical derivation). Here, it is seen that core similarity variables for similarity transformation of velocity field dominate the governing ordinary differential equations of vapour-gas mixture film flow.

\section{Conclusions}

For solving the governing partial differential equations, the similarity transformation of the equations is usually done. For such transformation, some dimensionless similarity variables (or parameters) are formed, and each similarity variable (or parameter) as the group of the dimensional physical variables to reduce the number of the independent variables of the physical phenomena. These similarity variables (or parameters) will simplify the governing partial differential equations for getting the solutions more easily.

Our innovative similarity transformation, was developed for in-depth research on convection hydrodynamics and heat and mass transfer, covering convection heat transfer, as well as heat and mass transfer of film boiling/condensation convection. Obviously, it covers very wide area of convection heat and mass transfer

Currently popular Fakner-Skan transformation on convection heat and mass transfer has some inconvenience for similarity transformation of the core variable on velocity field. By using this transformation, it is necessary to first induce flow function and group theory to derive an intermediate function for an indirect similarity transformation of the velocity field. It is a complicated process and allows difficult consideration of variable physical properties, and then confines the practical value of research results on convection heat and mass transfer.

Compared the Falkner-Skan transformation, a big advantage of our innovative similarity transformation lies in resolving the above core work. With our similarity transformation, the velocity variables can be directly transformed to the corresponding dimensionless similarity velocity variables. Then, all above inconvenient actions caused by using Falkner-Skan transformation can be avoided. In addition, our innovative similarity transformation can transform the variable physical properties into the physical property factors, organically combined with the transformed governing ordinary differential equations, and then simplify greatly the treatment of variable physical properties. It demonstrates that our innovative similarity transformation is a better alternative over the traditional Falkner-Skan transformation for in-depth research on convection heat and mass transfer. It was found that this innovative similarity transformation has wide application in engineering.

\section{Nomenclature}
$C_{m g}$
gas mass fraction in vapour-gas mixture
$C_{m v}$ vapor mass fraction in vapour-gas mixture
$C_{m v, \infty}$ bulk vapor mass fraction

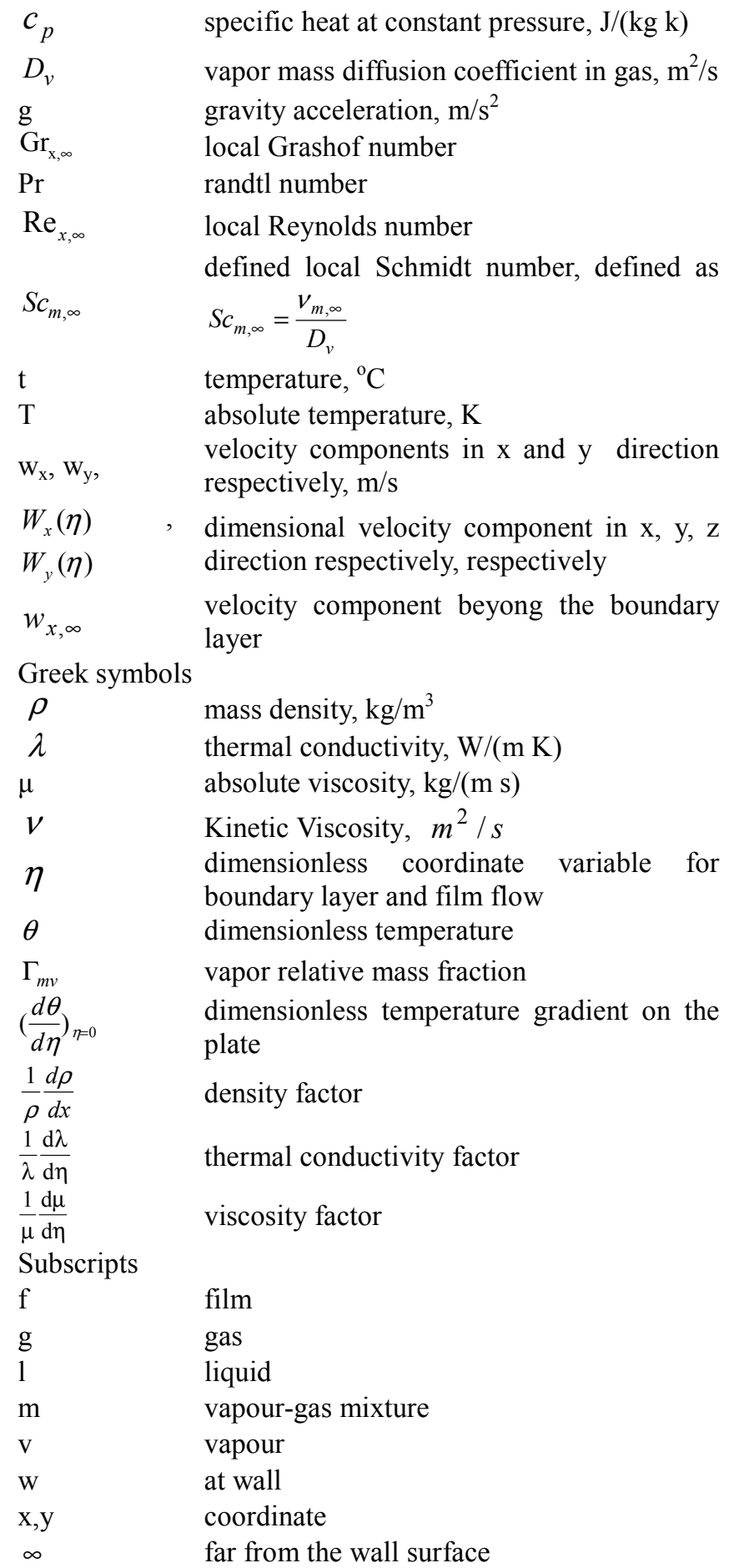

\section{References}

[1] L. Prandtl, ÜbDie Flussigkeitsbewegung bei Sehr Kleiner Reibung, Proc. 3d Intern. Math. Koug. Heidelberg, 1904.

[2] V.M. Falkner, S.W. Skan, Some approximate solutions of the boundary layer equations, Phil. 264 Mag. 12, pp.865, 1931.

[3] D.Y. Shang, "Theory of heat transfer with forced convection film flows", Series (book) of Heat and Mass Transfer, Springer-Verlag, Berlin, Heidelberg, New York, 1st ed., 346 p. 2011. 
[4] D.Y. Shang, " Free Convection Film Flows and Heat Transfer Models of Laminar Free Convection with Phase Change for Heat and Mass Transfer", Series (book) of Heat and Mass Transfer, Springer-Verlag, Berlin, Heidelberg, NewYork, 2nd ed., 535 p, 2013.

[5] D. Y. Shang and B. X. Wang, Effect of variable thermophysical properties on laminar free convection of gas, Int. J. Heat Mass Transfer, Vol.33, No.7, pp.1387-1395, 1990.

[6] D. Y. Shang and B. X. Wang, Effect of variable thermophysical properties on laminar free convection of polyatomic gas, Int. J. Heat Mass Transfer, Vol. 34, No.3, pp. 749-755, 1991.

[7] D.Y. Shang, B.X. Wang, Y. Wang and Y. Quan, Study on liquid laminar free convection with consideration of variable thermophysical properties, Int. J. Heat Mass transfer, Vol.36, No.14, pp.3411-3419, 1993.

[8] D. Y. Shang, B. X. Wang and L. C. Zhong, A study on laminar film boiling of liquid along an isothermal vertical plate in a pool with consideration of variable thermophysical properties, Int. J. Heat Mass Transfer, Vol.37, No.5, PP. 819-828, 1994.

[9] D.Y. Shang and T. Adamek, Study on laminar film condensation of saturated steam on a vertical flat plate for consideration of various physical factors including variable thermophysical properties, Warme-und Stoffübertragung 30,pp. 89-100, 1994.

[10] D.Y. Shang and B.X. Wang, An extended study on steadystate laminar film condensation of a superheated vapor on an isothermal vertical plate, Int. J. Heat Mass Transfer, Vol.40, No.4, pp.931-941, 1997.
[11] D.Y. Shang anf L.C. Zhong, Extensive study on laminar free film condensation from vapor-gas mixture, Int. J. Heat and mass Transfer, Vol.51, pp. 4300-4314, 2008.

[12] H. Andersson and D.Y. Shang, An extended study of hydrodynamics of gravity-driven film flow of power-law fluids Fluid Dynamics Research.pp.345- 357,1998.

[13] D.Y. Shang and H. I. Andersson , Heat transfer in gravitydriven film flow of power-law fluids, Int. J. Heat Mass Transfer 42, No.11, pp. 2085-2099, 1999.7.

[14] D.Y. Shang and J. Gu, Analyses of pseudo-similarity and boundary layer thickness for non-Newtonian falling film flow, Heat Mass Transfer, Vol. 41,No.1, pp. 44-50, 2004.

[15] G. Taton, T. Rok1,2, E. Rokita, Temperature distribution assessment during radiofrequency ablation , IFMBE Proceedings 22, pp. 2672-2676, 2008

[16] G. Taton, T. Rok, E. Rokita, Estimation of temperature distribution with the use of a thermo- camera, Pol J Med Phys Eng.,14(1), pp. 47-61, 2008.

[17] AA Turkin, M Dutka, D Vainchtein, S Gersen , etc., Deposition of $\mathrm{SiO} 2$ nanoparticles in heat exchanger during combustion of biogas, Applied Energy, Vol. 113, pp. 11411148,2014

[18] Y.Gao, Q.K.Liu, W.K.Chow, M.Wu, Analytical and experimental study on multiple fire sources in a kitchen, Fire Safety Journal 63, pp. 101-112, 2014. 